\title{
Canonical Transformation of Potential Model Hamiltonian Mechanics to Geometrical Form I
}

\author{
Yosef Strauss ${ }^{1}$, Lawrence P. Horwitz ${ }^{1,2,3,4}$, Jacob Levitan ${ }^{2} \mathbb{D}$ and Asher Yahalom ${ }^{5,6, *(\mathbb{D})}$ \\ 1 Department of Mathematics, Ben Gurion University of the Negev, Be'er Sheva 84105, Israel; \\ yossefst@ariel.ac.il (Y.S.); larry@tauex.tau.ac.il (L.P.H.) \\ 2 Department of Physics, Ariel University, Ariel 40700, Israel; levitan@ariel.ac.il \\ 3 Department of Physics, Tel Aviv University, Ramat Aviv 69978, Israel \\ 4 Department of Physics, Bar Ilan University, Ramat Gan 52900, Israel \\ 5 Department of Electrical \& Electronic Engineering, Ariel University, Ariel 40700, Israel \\ 6 PPPL, Princeton University, Princeton, NJ 08543, USA \\ * Correspondence: asya@ariel.ac.il
}

Received: 29 April 2020; Accepted: 10 June 2020; Published: 14 June 2020

\begin{abstract}
Using the methods of symplectic geometry, we establish the existence of a canonical transformation from potential model Hamiltonians of standard form in a Euclidean space to an equivalent geometrical form on a manifold, where the corresponding motions are along geodesic curves. The advantage of this representation is that it admits the computation of geodesic deviation as a test for local stability, shown in recent previous studies to be a very effective criterion for the stability of the orbits generated by the potential model Hamiltonian. We describe here an algorithm for finding the generating function for the canonical transformation and describe some of the properties of this mapping under local diffeomorphisms. We give a convergence proof for this algorithm for the one-dimensional case, and provide a precise geometric formulation of geodesic deviation which relates the stability of the motion in the geometric form to that of the Hamiltonian standard form. We apply our methods to a simple one-dimensional harmonic oscillator and conclude with a discussion of the relation of bounded domains in the two representations for which Morse theory would be applicable.
\end{abstract}

Keywords: classical Hamiltonian dynamics; symplectomorphism; geometric representation; geodesic deviation; stability

PACS: 02.40.Ry; 02.40.Yy; 45.20.Jj; 45.10.Na

\section{Introduction}

This paper is concerned with the development of a new method for embedding the motion generated by a classical Hamiltonian of standard form into a Hamiltonian defined by a bilinear form on momenta with coordinate-dependent coefficients (forming an invertible matrix) by means of a canonical transformation. This type of Hamiltonian, which we shall call geometric, by applying Hamilton's equations, results in equations of motion of geodesic form. The coefficients of the resulting bilinear form in velocities can be considered to be a connection form associated with the coefficients in the momenta in the geometric Hamiltonian considered as a metric on the corresponding coordinates. The advantage of this result, which may be considered to be an embedding of the motion induced by the original Hamiltonian into an auxiliary space for which the motion is governed by a geodesic structure, is that the deviation of geodesics on such a manifold (involving higher order derivatives than the usual Lyapunov criteria) can provide a very sensitive test of the stability of the original Hamiltonian motion. 
In previous work, an ad hoc construction of a geometrical embedding using a conformal metric [1] introduced. Casetti and Pettini [2] have investigated the application of the Jacobi metric and the extension of the analysis of the resulting Jacobi equations along a geodesic curve in terms of a parametric oscillator; such a procedure could be applied to the constuction we discuss here as well. The relation of the stability of geometric motions generated by metric models previously considered to those of the motion generated by the original Hamiltonian is generally, however, difficult to establish. The transformation that we shall construct here preserves a strong relation with the original motion due to its canonical structure.

The methods we shall use are fundamentally geometric, involving the properties of symplectic manifolds which enable the definition and construction of the canonical transformation without using the standard Lagrangian methods. These geometric methods provide a rigorous framework for this construction, which makes accessible a more complete understanding of the dynamics.

The theory of the stability of Hamiltonian dynamical systems has been discussed in depth, for example, in the books of Ar'nold [3], Guckenheimer and Holmes [4], and recently by DiBenedetto [5,6]. In his discussion of stability, Gutzwiller [7] (see also Miller and Curtiss [8]) discusses the example of a Hamiltonian of geometric type, where the Hamiltonian, instead of the standard expression

$$
H(q, p)=\frac{p^{2}}{2 m}+V(q)
$$

has the form (in two or more dimensions),

$$
H_{G}(x, \pi)=\frac{1}{2} g_{i j}(x) \pi^{i} \pi^{j}
$$

with indices summed (We use the convention, differing from that of the standard literature on differential geometry, of denoting coordinates with lower indices and momenta with upper indices, to conform with the usage in [1].). In one dimension, $g(x)$ would be just a scalar function, but, as we shall see, is still of interest. We shall call such a structure geometrical. We shall call the space of the standard variables $\{q, p\}$ the Hamilton space. The application of Hamilton's equations to Equation (2) results in a geodesic type equation

$$
\ddot{x}_{\ell}=-\Gamma_{\ell}^{m n} \dot{x}_{m} \dot{x}_{n}
$$

where the coefficients have the structure of a connection form (here, $g^{i j}$ is the inverse of $g_{i j}$ )

$$
\Gamma_{\ell}^{m n}=\frac{1}{2} g_{\ell k}\left(\frac{\partial g^{k m}}{\partial x_{n}}+\frac{\partial g^{k n}}{\partial x_{m}}-\frac{\partial g^{n m}}{\partial x_{k}}\right)
$$

This connection form is compatible with the metric $g_{i j}(x)$ by construction, i.e., the covariant derivative of of $g_{i j}$ constructed with the $\Gamma_{\ell}^{m n}$ of Equation (3) vanishes, and we recognize that the dynamics generated on the coordinates $\{x\}$ is a geodesic flow. It can carry, moreover, a tensor structure which may be inferred from the requirement of invariance of the form given in Equation (2) under local coordinate transformations.

The stability of such a system may be tested by studying the geodesic deviation, i.e., by studying what happens when one shifts to a nearby geodesic curve, corresponding to a local change in initial conditions. The resulting separation of the two geodesic curves provides a very sensitive test of stability (see Gutzwiller [7], and for its application to general relativity, Weinberg [9]). An exponentially growing deviation is characteristic of local instability, and may lead to chaotic behavior of the global motion.

In order to obtain a criterion in the case of a standard Hamiltonian of the form given in Equation (1), Horwitz et al. [1] constructed an ad hoc transformation of this Hamiltonian to a Hamiltonian of the form of (2) by defining the metric as

$$
g_{\ell k}(x)=\delta_{\ell k} \phi(x)
$$


where (with a relation between $x$ and $q$ to be explained below)

$$
\phi(x)=\frac{E}{E-V(q)} \equiv F(q),
$$

and $E$ is taken to be the assumed common (conserved) value of $H$ and $H_{G}$.

The motion induced on the coordinates $\{x\}$ by $H_{G}$, after the local tangent space transformation $\dot{y}^{k}=g^{k \ell}(x) \dot{x}_{\ell}$, results in a geometric embedding of the original Hamiltonian motion for which the geodesic deviation gives a sensitive diagnostic criterion for the stability of the original Hamiltonian motion [1,10-13]. The condition of dynamical equivalence of the two systems, based on enforcing equal values of the momenta at all times (the transformation is not necessarily canonical), provides a constraint that establishes a correspondence between the coordinatizations $\{x\}$ and $\{q\}$ in the sense that $\phi(x)$ can be expressed as a series expansion in $F(q)$ and its derivatives, and conversely, $F(q)$ can be expressed as a series expansion in $\phi(x)$ and its derivatives, in a common domain of analyticity [14]; in this way, all derivatives of $\phi(x)$ can be expressed in terms of derivatives of $F(q)$, and conversely.

The remarkable success of this method has not yet been explained, although some insights were provided in [15]. In the theory of symplectic manifolds (see, for example [16]), a well-defined mechanism exists for transforming a Hamiltonian of the form of Equation (1) to that of Equation (2) (with a possibly conformal metric) by a rigorous canonical transformation, admitting the use of geodesic deviation to determine stability, which would then be clearly associated with the original Hamiltonian motion. We shall define this theory, and describe some of its properties, in this paper.

We remark that in an analysis [17] of the geodesic deviation treated as a parametric oscillator, a procedure of second quantization was carried out providing an interpretation of excitation modes for the instability in a "medium" represented by the background Hamiltonian motion. This interpretation would be applicable to the results of the construction we present here as well.

In the following, we describe this mapping and an algorithm for obtaining solutions. We give a convergence proof for the recurrence relations for the generating function in the one-dimensional case which appears to be applicable to the general $n$-dimensional case. Although the algorithm for the construction is clearly effective (and convergent), its realization requires considerable computation for specific applications, which we shall carry out in succeeding publications. The resulting programs could then be applied to a wide class of systems to provide stability criteria without exhaustive simulation; the local criteria to be developed could, furthermore, be used for the control of intrinsically chaotic systems [13].

In this paper we discuss some general properties of the framework. In Section 2, we give the basic mathematical methods in terms of the geometry of symplectic manifolds.

A central motivation for our construction is to make available the study of stability by means of geodesic deviation. This procedure is studied in Section 3, in terms of geometric methods, making clear the relation between stability in the geometric manifold and the original Hamiltonian motion.

In Section 4, an algorithm is described for solving the nonlinear equations for the generating function of the canonical transformation. In Section 5, we study this algorithm for the one-dimensional case, and prove convergence of the series expansions, under certain assumptions in Section 6. The series expansions that we obtain can be studied by methods of Fourier series representations; the nonlinearity leads to convolutions of analytic functions (see, for example, Hille [18]) that may offer approximation methods that could be useful in studying specific cases. We plan to discuss this topic in a future publication.

Since the iterative expansions for the generating function could be expected to have only bounded domains of convergence, we consider, in Section 7, the possibility of shifting the origin of the expansion in general dimension, As for the analytic continuation of a function of a complex variable, this procedure can extend the definition of the generating function to a maximal domain.

Since the image space of the symplectomorphism has geometrical structure, it is natural to study its properties under local diffeomorphisms. A local change of variable alters the structure of 
the symplectomorphism. We study the effect of such diffeomorphims on the generating function (holding the original Euclidean variables fixed) in Section 8.

Further mathematical implications, such as relations to Morse theory (e.g., $[19,20]$ ), are briefly discussed in Section 9; a more extended development of this topic will be given in a succeeding publication.

\section{Basic Mathematical Formulation}

The notion of a symplectic geometry is well-known in analytic mechanics through the existence of the Poisson bracket of Hamilton-Lagrange mechanics, i.e., for $A, B$ functions of the canonical variables $q, p$ on phase space, the Poisson bracket is defined by

$$
\{A, B\}_{P B}=\sum_{k}\left(\frac{\partial A}{\partial q_{k}} \frac{\partial B}{\partial p^{k}}-\frac{\partial B}{\partial q_{k}} \frac{\partial A}{\partial p^{k}}\right)
$$

The antisymmetric bilinear form of this expression has the symmetry of the symplectic group, associated with the symmetry of the bilinear form $\xi_{i} \eta^{i j} \xi_{j}$, with $i, j=1,2, \ldots 2 n$ and $\eta^{i j}$ an antisymmetric matrix (independent of $\xi$ ); the $\left\{q_{k}\right\}$ and $\left\{p^{k}\right\}$ can be considered as the coordinatization of a symplectic manifold.

The coordinatization and canonical mapping of a symplectic manifold [16], to be called a symplectomorphism, can be constructed by considering two $n$-dimensional manifolds $X_{1}$ and $X_{2}$ (to be identified with the target and image spaces of the map) with associated cotangent bundles $M_{1}=T^{*} X_{1}, M_{2}=T^{*} X_{2}$, so that

$$
M_{1} \times M_{2}=T^{*} X_{1} \times T^{*} X_{2} \simeq T^{*}\left(X_{1} \times X_{2}\right) .
$$

To complete the construction of the symplectomorphism, one defines the involution $\sigma_{2}$. The action of this involution, in terms of the familiar designation, if $\left(x_{2}, p_{2}\right) \in M_{2}=T^{*} X_{2}$ is a point in $M_{2}$ (so that $x_{2}$ is a point in $X_{2}$ and $p_{2}$ is a one-form at the point $\left.x_{2}\right)$, we define

$$
\sigma_{2}\left(x_{2}, p_{2}\right)=\left(x_{2},-p_{2}\right)
$$

We then define

$$
\sigma=\mathrm{id}_{M_{1}} \times \sigma_{2}
$$

where id $M_{M_{1}}$ is the identity map on $M_{1}$.

This construction can be extended to a coordinate patch on $M_{2}$, enabling the construction of a bilinear form in the tangent space of $M_{2}$. A vector

$$
v=v^{j} \frac{\partial}{\partial u^{j}}
$$

where, on some coordinate patch on $M_{2}$ with $u_{j}=x_{2}{ }^{j}, j=1 \ldots n$, and $u^{j}=p_{2, j-n}, j=n+1, \ldots 2 n$, and $\tilde{u}=\sigma_{2} u$, in the tangent space $T M_{2}$, gives rise to a one-form; the differential of the map induced by $\sigma_{2}$ results in the vector ("pushforward"),

$$
d \sigma_{2}(v)=v^{j} \frac{\partial \tilde{u}^{i}}{\partial u^{j}} \frac{\partial}{\partial \tilde{u}^{i}} .
$$

If $\beta$ is a one-form, the ("pullback") map $\sigma_{2}{ }^{*}: T^{*} M_{2} \rightarrow T^{*} M_{2}$, defined by

$$
\sigma_{2}^{*} \beta(v)=\beta\left(d \sigma_{2}(v)\right) .
$$

provides the characteristic antisymmetric form on the symplectic manifold required for the formulation of Lagrangian mechanics. 
One then proceeds to define a smooth function $f \in C^{\infty}\left(X_{1} \times X_{2}\right)$; if $d f$ is a closed 1-form on $T\left(X_{1} \times X_{2}\right)$, call

$$
Y_{f}=\left\{\left((x, y),(d f)_{(x, y)}\right):(x, y) \in X_{1} \times X_{2}\right\}
$$

Then,

$$
\left.Y_{f}^{\sigma}=\sigma\left(Y_{f}\right)=\left\{\left((x, y), d_{x} f,-d_{y} f\right)\right):(x, y) \in X_{1} \times X_{2}\right\} .
$$

If $Y_{f}^{\sigma}$ is a graph of a diffeomorphism $\varphi: M_{1} \rightarrow M_{2}$, then $\varphi$ is a symplectomorphism. Now suppose $\varphi: M_{1}-T^{*} X_{1} \rightarrow M_{2}=T^{*} X_{2}$ is the map

$$
\varphi(x, \xi)=(y, \eta)
$$

and $Y_{f}^{\sigma}$ is its graph, then

$$
\begin{aligned}
t \xi_{i} d x^{i} & =\quad \frac{\partial f}{\partial x^{i}} d x^{i} \Rightarrow \xi_{i}=\frac{\partial f}{\partial x^{i}} \\
\eta_{i} d y^{i} & =-\frac{\partial f}{\partial y^{i}} d y^{i} \Rightarrow \eta_{i}=-\frac{\partial f}{\partial y^{i}} .
\end{aligned}
$$

We may now attempt to solve (16) to obtain

$$
y=y(x, \xi)
$$

and then the second of (17) to obtain

$$
\eta=\eta(x, y(x, \xi)) \equiv \eta(x, \xi)
$$

and with this, determine the symplectomorphism

$$
\varphi(x, \xi)=(y(x, \xi), \eta(x, \xi)) .
$$

In its application to Hamiltonian mechanics, in the usual notation, let

$$
\varphi\left(q^{1}, \ldots q^{n}, p_{1}, \ldots p_{n}\right)=\left(x^{1}, \ldots x^{n}, \pi_{1} \ldots \pi_{n}\right)
$$

between $M_{1}=T^{*} X_{1}$ and $M_{2}=T^{*} X_{2}$ through the equations

$$
\begin{aligned}
p_{i} & =\frac{\partial f(q, x)}{\partial q^{i}} \\
\pi_{i} & =-\frac{\partial f(q, x)}{\partial x^{i}}, \quad i=1,2 \ldots n,
\end{aligned}
$$

where we have denoted the generating function of the symplectomorphism $\varphi$ by $f$. We remark that the possibility of solving Equation (17) locally to obtain Equation (18) and Equation (19) requires that

$$
\operatorname{det}\left(\frac{\partial^{2} f(q, x)}{\partial q^{i} \partial x^{j}}\right) \neq 0
$$

The Equation (22), of the form of the usual canonical transformation derived by adding a total derivative to the Lagrangian in Hamilton-Lagrange mechanics, have been obtained here by a more general and more powerful geometric procedure (the theory of symplectomorphisms), enabling, as we shall see, a simple formulation of the transformation from the standard Hamiltonian form to a geometrical type Hamiltonian. 


\section{Geodesic Deviation}

The principal reason for introducing the canonical transformation from Hamiltonian form to the geometric form, as we have pointed out in the introduction, is to make accessible the very sensitive measure of stability provided by geodesic deviation. In this section we develop a geometrical formulation of this technique which makes clear the relation between stability in the geometric space and stability in the original Hamiltonian space.

Returning to the geometrical framework defined in Section 2, let $\mathbf{X}$ be a Hamiltonian vector field in the phase space $M_{1}$, satisfying

$$
i_{\mathbf{X}} \omega=d H,
$$

where $\omega$ is the canonical symplectic form on $M_{1}$. The integral curves of $\mathbf{X}$, obtained by solving Hamilton's equations for $H$, are the trajectories of the Hamilton dynamical system. Since the mapping $\varphi$ to $M_{2}$ is a symplectomorphism, the pullback by $\varphi$ of the canonical symplectic form $\tilde{\omega}$ on $M_{2}$ satisfies

$$
\varphi^{*} \tilde{\omega}=\omega
$$

If $d \varphi: T M_{1} \mapsto T M_{2}$ is the differential of $\varphi$ and we define the vector field $\mathbf{X}_{\text {geo }}=d \varphi(\mathbf{X})$, we have

$$
i_{\mathbf{X}_{g e o}} \tilde{\omega}=d H_{g e o},
$$

so that $\mathbf{X}_{g e o}$ is a Hamiltonian vector field in $T M_{2}$ with respect to the Hamiltonian function $H_{g e o}$; the integral curves for $\mathbf{X}_{\text {geo }}$ correspond to geodesics in $M$. We shall refer to such integral curves of $\mathbf{X}_{\text {geo }}$ as $M_{2}$ geodesics, or cotangent bundle geodesics.

Let $\gamma \subset M_{1}$ be a trajectory in phase space of the original dynamical system. Then, $\gamma^{\varphi}=\varphi(\gamma)$ is an $M_{2}$ geodesic. If $\tilde{\pi}: M_{2} \rightarrow M$ is the projection of the cotangent bundle $M_{2}=T^{*} M$ on the base manifold $M$, then $\tilde{\pi}\left(\gamma^{\varphi}\right)$ is a geodesic in $M$. For $G$ the map of the tangent bundle $M_{3}=T M$ to the cotangent bundle $M_{2}$, we apply the inverse map $G^{-1}: M_{2} \mapsto M_{3}$, the tangent bundle for $M$, i.e., $(x, \mathbf{v})$, where $\mathbf{v} \in T_{x} M$ ( $x$ is a point in $M$ ), to $\gamma^{\varphi}$, we obtain an $M_{3}$ (or tangent bundle) geodesic

$$
\gamma^{Q}=G^{-1}\left(\gamma^{\varphi}\right)=\left(G^{-1} \circ \varphi\right) \gamma=Q(\gamma)
$$

If now $\pi: M_{3} \mapsto M$ is the projection of the tangent bundle on the base manifold $M$, then $\pi\left(\gamma^{Q}\right)=\tilde{\pi}\left(\gamma^{\varphi}\right)$ is a geodesic in $M$. This establishes the equivalence of trajectories in the original Hamiltonian space with geodesics in the geometric space.

Let $u_{0} \in M_{1}$ be a point in phase space and let $\gamma_{0} \subset M_{1}$ be the curve given by $\gamma_{0}(t)=\phi_{t}\left(u_{0}\right)$, where $\phi_{t}$ is the flow in the phase space $M_{1}$ of the Hamiltonian dynamical system generated by $H$, i.e., $\gamma_{0}$ is a trajectory of the system such that $\gamma_{0}(0)=u_{0}$. Let $\tilde{W}^{2 n-1} \subset M_{1}$ be a surface of section at $u_{0}$, i.e., a hypersurface in $M_{1}$ transverse to the trajectories of the dynamical system and defined in some open neighborhood of $u_{0}$. Let $E_{0} \subset M_{1}$ be an equal energy hypersurface passing through a point $p_{0} \in E_{0}$, for which $d H=0$ on $E_{0}$, and let $W=W^{2 n-1} \cap E_{0}$. Then $W$ is a $2 n$-2-dimensional submanifold of $M_{1}$ such that the Hamiltonian $H$ has the same value at all points $u \in W$ and such that the trajectories of the dynamical system are transverse to $W$ at all points of intersection. Now, let $u$ be an arbitrary point in $W$; then it is a base point of a trajectory $\gamma_{u}$ given by $\gamma_{u}(t)=\phi_{t}(u)$. In a time interval $0 \leq t \leq T(T>0)$ we define a submanifold $N_{u_{0}} \subset M_{1}$ by

$$
N_{u_{0}}=\left\{\phi_{t}(u): \forall u \in W, \forall t \in[0, T]\right\} .
$$

Then, $N_{u_{0}}$ is parametrized by $(u, t)$, for $u \in W, t \in[0, T]$. and consists of trajectories of the dynamical system corresponding to all initial points $u \in W$. Now apply the mapping $Q$ to obtain a submanifold $N_{u_{0}} \mathrm{Q} \subset M_{1}$ according to

$$
N_{u_{0}}^{Q}=Q\left(N_{u_{0}}\right)=\left\{Q\left[\phi_{t}(u)\right]: \forall u \in W, \forall t \in[0, T]\right\} .
$$


Again, by construction, $N_{u_{0}} Q$ is parametrized by $(u, t)$, for $u \in W, t \in[0, T]$. For each $u \in W$, the curve $\gamma_{u} Q=Q\left(\gamma_{u}\right)$ is an $M_{3}$ geodesic curve given by $\gamma_{u} Q(t)=Q\left[\phi_{t}(u)\right]$ and $N_{u_{0}}{ }^{Q}$ consists of all such geodesic curves corresponding to all possible initial points $u \in W$. In particular, $\gamma_{0} Q=Q\left(\gamma_{0}\right)$ is the $M_{3}$ geodesic corresponding to to the trajectory $\gamma_{0}$ of the original dynamical system.

To calculate geodesic deviation, we now consider variations of such trajectories. Let $\gamma_{v a r} \subset W$ be a curve parametrized by a parameter $\alpha$ and based at the point $u_{0} \in W$. For some interval $I \subset \mathbf{R}$, with $0 \in I, \gamma_{v a r}$ is given by a smooth function $u(\alpha) \in W, \forall \alpha \in I$ and $u(0)=u_{0}$. The curve $\gamma_{v a r}$ corresponds to a two dimensional surface $S_{\text {var }}\left(\gamma_{v a r}\right) \subset N_{u_{0}}$ through the definition

$$
S_{\text {var }}\left(\gamma_{\text {var }}\right)=\left\{\phi_{t}(u(\alpha)): \alpha \in I, t \in[0, T]\right\}
$$

By construction, $(t, \alpha), t \in[0, T], \alpha \in I$ are coordinates on $S_{v a r}\left(\gamma_{v a r}\right)$, the variational surface of $\gamma_{0}$ corresponding to $\gamma_{v a r}$. Each such curve $\gamma_{U(\alpha)}$, given by $\gamma_{v a r}(t)=\phi_{t}(u(\alpha), t \in[0, T]$, is a trajectory of the original Hamiltonian system. Furthermore, $\gamma_{v a r}$ is carried by the flow $\phi_{t}$ to a variation curve $\gamma_{v a r}^{t}$ at time $t$ defined by $\gamma_{v a r}^{t}=\phi_{t}\left(\gamma_{v a r}\right)$, given explicitly by the function $\phi_{t}(\alpha)=\phi_{t}(u(\alpha))$, where $u(\alpha)$ is the function defining $\gamma_{v a r}^{t}$. Applying the mapping $Q$ to $S_{v a r}\left(\gamma_{v a r}\right)$, we obtain an $n$-1-dimensional surface in $M_{3}$ (two-dimensional surface in a three-dimensional problem)

$$
\begin{aligned}
S_{\text {var }}\left(\gamma_{\text {var }}\right)=Q\left[S_{\text {var }}\left(\gamma_{\text {var }}\right)\right]=\left\{\gamma_{u(\alpha)}^{Q}: \alpha \in I\right\} & =\left\{Q\left(\gamma_{u(\alpha)}\right): \alpha \in I\right\}= \\
& =\left\{Q\left[\phi_{t}(u(\alpha))\right]: \alpha \in I, t \in[0, T]\right\},
\end{aligned}
$$

where

$$
\gamma_{u(\alpha)}^{Q}=Q\left(\gamma_{u(\alpha)}\right)=Q\left[\phi_{t}(u(\alpha))\right]
$$

Note that $(t, \alpha), \quad t \in[0, T], \alpha \in I$ are coordinates on $S Q_{v a r}\left(\gamma_{v a r}\right)$, and that, since each curve $\gamma_{u(\alpha)}$ is a trajectory of the original dynamical system, $\gamma_{u(\alpha)}^{Q}$ is an $M_{3}$ geodesic. Therefore, $S^{Q}$ var $\left(\gamma_{v a r}\right)$ is a surface of variation for $\gamma_{0}{ }^{Q}$ consisting of $M_{3}$ geodesics. Furthermore, $\gamma_{v a r}{ }^{Q}, t=Q\left(\gamma_{v a r}{ }^{t}=\left[\phi_{t}\left(\gamma_{v a r}\right)\right]\right.$ is the variation at time $t$ in $S^{Q}{ }_{v a r}\left(\gamma_{v a r}\right)$ corresponding to the variation curve $\gamma_{v a r}{ }^{t} \subset S_{\text {var }}\left(\gamma_{v a r}\right)$. A parametrization of $\gamma_{v a r} Q, t$ is provided by the function $\gamma_{v a r} Q, t(\alpha)=\gamma_{u(\alpha)} Q(t), \alpha \in I$, with $t$ constant.

We now wish to investigate the deviation of nearby trajectories of the original Hamiltonian system by considering the deviation of the corresponding geodesics in $M_{3}$. We quantify the deviation of nearby trajectories from the base trajectory $\gamma_{0}$ in $N_{u_{0}}$, i.e., on the variational surface $S_{v a r}\left(\gamma_{v a r}\right)$, by studying the evolution along $\gamma_{0}$ of the tangent vector to the variation curve $\gamma_{v a r}{ }^{t}$. The tangent vector, which we call the phase space trajectory deviation vector is formally given by

$$
\mathbf{V}_{t r j}(t)=\left.\left[\frac{\partial}{\partial \alpha} \gamma_{v a r}{ }^{t}(\alpha)\right]\right|_{\alpha=0}=\left.\left[\frac{\partial}{\partial \alpha} \phi_{t}(u(\alpha))\right]\right|_{\alpha=0}, \quad \mathbf{V}_{t r j}(t) \in T M_{1}
$$

The deviation vector $\mathbf{V}_{t r j}(t)$ is mapped by the differential $d Q_{*}$ of the mapping $Q$ into a deviation vector in $T M_{3}$, formally given by:

$$
\begin{aligned}
& \mathbf{J}_{d e v}(t)=\left[\frac{\partial}{\partial \alpha} \gamma_{v a r} Q, t(\alpha)\right]_{\alpha=0}=\left[\frac{\partial}{\partial \alpha} \gamma_{u(\alpha)} Q(t)\right]_{\alpha=0}=\left[\frac{\partial}{\partial \alpha} Q\left[\phi_{t}(u(\alpha))\right]\right]_{\alpha=0}= \\
& d Q_{*}\left(\left[\frac{\partial}{\partial \alpha} \phi_{t}(u(\alpha))\right]_{\alpha=0}\right)=d Q_{*}\left(\mathbf{V}_{t r j}(t)\right), \quad \mathbf{J}_{d e v}(t) \in T M_{3},
\end{aligned}
$$

where $d Q: T M_{1} \mapsto T M_{3}$ is the differential of the map $Q$.

In order to obtain a more explicit expression for $\mathbf{J}_{d e v}(t)$ we will need a more explicit expression for the points in $N^{Q} u_{0} \subset M_{3}$ and, in particular, points in $S^{Q_{v a r}}\left(\gamma_{v a r}\right)$. Recall the fact that $(t, \alpha), t \in[0, T], \alpha \in I$ serve as coordinates in $N^{Q_{u_{0}}}$. The point corresponding to the pair $(t, \alpha)$ is $\gamma_{u(\alpha)} Q(t)=Q\left[\phi_{t}(u(\alpha))\right]=(x(x, \alpha), \mathbf{T}(t, \alpha))$, where $x(t, \alpha)=\pi\left(\gamma_{u, \alpha}^{Q}\right) \in M$ is a point on the 
geodesic $\pi\left(\gamma_{u(\alpha)} Q(t)\right)$ at the point $x(t, \alpha)$. Since $\mathbf{T}(t, \alpha)$ forms a vector field defined on $\pi\left(N^{Q} u_{0}\right)$ and, in particular, along the geodesic curve $\gamma^{Q, t}{ }_{v a r}$, its $\alpha$ derivative is given by the covariant derivative $\frac{\nabla \mathbf{T}(t, \alpha)}{\partial \alpha}$. Then, we find that

$$
\mathbf{J}_{d e v}(t)=\left[\frac{\partial}{\partial \alpha} Q\left[\phi_{t}(u(\alpha))\right]\right]_{\alpha=0}=\left(\left.\left.\frac{\partial x(t, \alpha)}{\partial \alpha}\right|_{\alpha=0} \frac{\nabla \mathbf{T}(t, \alpha)}{\partial \alpha}\right|_{\alpha=0}\right)^{T} .
$$

Note that $\mathbf{J}_{d e v}(t) \in T_{x(t, 0)} M \oplus T_{x(t, 0)} M=T M_{3}$.

The standard definition of the geodesic deviation vector for geodesics in $M$ is

$$
\mathbf{J}(t)=\left.\left(\frac{\partial x(t, \alpha)}{\partial \alpha}\right)\right|_{\alpha=0}, \quad \mathbf{J}(t) \in T_{x(t, 0)} M .
$$

According to Theorem 10 of Frankel [19],

$$
\frac{\nabla \mathbf{J}(t)}{\partial t}=\left.\left(\frac{\nabla \mathbf{T}(t, \alpha)}{\partial \alpha}\right)\right|_{\alpha=0},
$$

so that

$$
\mathbf{J}_{\text {dev }}(t)=\left(\mathbf{J}(t), \frac{\nabla \mathbf{J}(t)}{\partial t}\right)^{T}
$$

where $t$ is the affine parameter parametrizing $\gamma_{0}^{Q}$.

The equation of evolution of $\mathbf{J}_{d e v}(t)$, i.e., the dynamical system representation of the geodesic deviation equation, has been studied in ref. [17].

Let $\mathbf{X}, \mathbf{Y}, \mathbf{Z} \in T_{p} M$ be $\left(n=\right.$ dimensional) vectors and let $R_{p}(\mathbf{X}, \mathbf{Y}): T_{p} M \mapsto T_{p} M$ be the curvature transformation at the point $p \in M$ i.e., the linear transformation with matrix elements $\left[R_{p}(\mathbf{X}, \mathbf{Y})\right]_{j}{ }^{i}=R_{j k \ell}^{i} X^{i} Y^{j}$ so that

$$
R_{p}(\mathbf{X}, \mathbf{Y}) \mathbf{Z}=\left(R_{j k \ell}^{i} X^{k} Y^{\ell} Z^{j}\right) \partial_{i}
$$

where $\partial_{i}$ are coordinate vectors at $p$ and $\left(X^{k}, Y^{k}, Z^{k}, 1 \leq k \leq n\right.$ are the components of $\mathbf{X}, \mathbf{Y}, \mathbf{Z}$ with respect to the basis $\left\{\partial_{k}\right\}_{k=1}{ }^{n}$ ). The quantities $R^{i}{ }_{j k \ell}$ are the components of the Riemann curvature tensor at the point $p$.

Furthermore, if $\langle\cdot, \cdot\rangle_{T_{p} M}$ denotes the inner product defined on $T_{p} M$ with the metric $g(\cdot, \cdot)$ on $M$, then for $\mathbf{W} \in T_{p} M$ we have

$$
<R_{p}(\mathbf{X}, \mathbf{Y}) \mathbf{Z}, \mathbf{W}>_{T_{p} M}=R_{j k \ell}^{i} X^{k} Y^{\ell} Z^{j} W_{i}
$$

where $W_{i}=g_{i j} W^{j}$. For the geodesic $\gamma_{0}{ }^{Q} \in M$, given in terms of the function $\gamma_{0}{ }^{Q}(t)=Q\left[\phi_{t}\left(u_{0}\right)\right]$, using the above notation for the curvature transformation, the geodesic deviation equation along $\gamma_{0}{ }^{Q}$ is

$$
\frac{\nabla^{2} \mathbf{J}(t)}{d t^{2}}+R_{\gamma_{0} Q(t)}(\mathbf{J}(t), \mathbf{T}(t))(\mathbf{T}(t))=0,
$$

where $\mathbf{J}(t)$ is the geodesic deviation vector defined above, $\mathbf{T}(t) \equiv \mathbf{T}_{\gamma_{0} Q(t)}$ is the tangent vector to $\gamma_{0} Q$ at the point $\gamma_{0} Q(t)$ and $R_{\gamma_{0} Q(t)}$ is the curvature tensor at the point $\gamma_{0} Q(t)$. The dynamical system representation of the geodesic deviation equation corresponds to putting Equation (41) into the form

$$
\frac{\nabla}{d t}\left(\begin{array}{c}
\mathbf{J}(t) \\
\frac{\nabla \mathbf{J}(t)}{d t}
\end{array}\right)=\left(\begin{array}{cc}
0 & I \\
-R_{\gamma_{0} Q(t)}(\cdot, \mathbf{T}(t)) \mathbf{T}(t) & 0
\end{array}\right)\left(\begin{array}{c}
\mathbf{J}(t) \\
\frac{\nabla \mathbf{J}(t)}{d t}
\end{array}\right) .
$$


Denoting

$$
\hat{R}_{\gamma_{0}^{Q}(t)}=\left(\begin{array}{cc}
0 & I \\
-R_{\gamma_{0} Q(t)}(\cdot, \mathbf{T}(t)) \mathbf{T}(t) & 0
\end{array}\right)
$$

and using Equation (38), we may write Equation (42) in the shorter form

$$
\frac{\nabla \mathbf{J}_{d e v}}{d t}=\hat{R}_{\gamma_{0} Q(t)} \mathbf{J}_{d e v} .
$$

The behavior of the solution $\mathbf{J}_{d e v}$ of Equation (44) determines the deviation properties of geodesics near $\gamma_{0}{ }^{Q}$ as a function of $t$ and, through the relation $\mathbf{V}_{t r j}(t)=d Q^{-1}\left(\mathbf{J}_{d e v}(t)\right)$ obtained from Equation (34), also the deviation of trajectories of the original dynamical system near $\gamma_{0}$ over time. The deviation of trajectories of the original system near $\gamma_{0}$ is therefore governed by the curvature transformation $R_{\gamma_{0} Q(\cdot)}$ along the geodesic $\gamma_{0} Q_{(\cdot) \text {. }}$

\section{Formulation of the Algorithm}

The purpose of the canonical transformation we have discussed above is to construct a Hamiltonian of the geometrical form of Equation (2) by means of a canonical transformation from a Hamiltonian of the form of Equation (1). As above, we label the coordinates and momenta of the image space by $\left\{x_{i}\right\}$ and $\left\{\pi^{i}\right\}$ (we do not require that $p^{i}$ and $\pi^{i}$ are necessarily simply related for all $t$ here; the equivalence of the dynamics is assured by the canonical nature of the transformation). We must therefore find the generating function $f(q, x)$ and the metric $g_{i j}(x)$ from the statement

$$
\frac{p^{2}}{2 m}+V(q)=\frac{1}{2 m} g_{i j}(x) \pi^{i} \pi^{j}
$$

Substituting Equation (22) for the momenta, the problem is to solve (note that the left hand side treats the indices as Euclidean since it does not carry the local coordinate transformations available to the geometric form on the right hand side)

$$
V(q)+\frac{1}{2 m}\left(\frac{\partial f(q, x)}{\partial q_{i}}\right)\left(\frac{\partial f(q, x)}{\partial q_{i}}\right)=\frac{1}{2 m} g_{i j}(x)\left(\frac{\partial f(q, x)}{\partial x_{i}}\right)\left(\frac{\partial f(q, x)}{\partial x_{j}}\right) .
$$

Assuming analyticity in the neighborhood of the origin of the coordinates $\{q\}$, and in the potential term $V(q)$, one can write a power series expansion of the generating function and the potential, and identify the resulting powers of $q_{i}, q_{j} \ldots$ and their products. This procedure provides an effective recursive algorithm for a system of nonlinear first order equations in the expansion coefficients since the powers of $q$ on the right hand side occurring in the expansion of $f(q, x)$ are higher by one order that the expansions on the left hand side, which contain derivatives with respect to $q$. Assuming analyticity in $\{x\}$ as well near the origin (as for Riemann normal coordinates), one can find a recursion relation for the resulting coefficients.

For example, in two dimensions, one may expand, into some radius of convergence,

$$
f\left(q^{1}, q^{2}, x^{1}, x^{2}\right)=\sum_{k, \ell=0}^{\infty} C_{k, \ell}\left(x^{1}, x^{2}\right)\left(q^{1}\right)^{k}\left(q^{2}\right)^{\ell}
$$

and expand $V\left(q^{1}, q^{2}\right)$ in power series

$$
V\left(q^{1}, q^{2}\right)=\Sigma_{k, \ell=0}^{\infty} v_{k, \ell}\left(q^{1}\right)^{k}\left(q^{2}\right)^{\ell} .
$$


Substituting into the relation, Equation (46) (in teh two-dimensional form), and equating coefficients of powers of $q^{1}$ and $q^{2}$, one finds the following recursion relations:

$$
\begin{aligned}
v_{k, \ell}+\Sigma_{k=0}^{m} \Sigma_{\ell=0}^{n}\left[(k+1)(m-k+1) C_{(k+1), \ell}\left(x^{1}, x^{2}\right) C_{(m-k+1),(n-1)}\left(x^{1}, x^{2}\right)\right. \\
\left.+(\ell+1)(n-\ell+1) C_{k,(\ell+1)}\left(x^{1}, x^{2}\right) C_{(m-k),(n-\ell+1)}\left(x^{1}, x^{2}\right)+2 v_{n, m}\right]= \\
\quad=\Sigma_{k=0}^{m} \Sigma_{\ell=0}^{n}\left[g^{11}\left(x^{1}, x^{2}\right) \frac{\partial C_{k, \ell}}{\partial x^{1}}\left(x^{1}, x^{2}\right) \frac{\partial C_{m-k, n-1}}{\partial x^{1}}\left(x^{1}, x^{2}\right)\right. \\
+2 g^{12}\left(x^{1}, x^{2}\right) \frac{\partial C_{k, \ell}}{\partial x^{1}}\left(x^{1}, x^{2}\right) \frac{\partial C_{m-k, n-1}}{\partial x^{2}}\left(x^{1}, x^{2}\right) \\
\left.+g^{22}\left(x^{1}, x^{2}\right) \frac{\partial C_{k, \ell}}{\partial x^{2}}\left(x^{1}, x^{2}\right) \frac{\partial C_{m-k, n-1}}{\partial x^{2}}\left(x^{1}, x^{2}\right)\right] .
\end{aligned}
$$

The solution of this system of equations, for a given potential $V$ requires, even in two dimensions, significant computational power. Our initial investigations indicate reasonable behavior, with strong indications of convergence, for some simple cases.

Although the physically interesting cases are in two or more dimensions, where curvature generated by the geometric Hamiltonian plays an important role in the formation of geodesic curves and for many practical problems, we shall describe the general structure of the calculation in one dimension below as well as to give a convergence proof for this case, which, it appears, can be extended to arbitrary dimension. Some basic properties of the higher-dimensional structure are discussed below as well, but a full development of the algorithm in higher dimensions and applications will be treated in succeeding publications.

\section{Study of the One-Dimensional Case}

In one dimension, Equation (46) becomes

$$
\frac{1}{2 m}\left(\frac{\partial f(q, x)}{\partial q}\right)^{2}+V(q)=\frac{1}{2 m} g(x)\left(\frac{\partial f(q, x)}{\partial x}\right)^{2}
$$

The recursion relation for the one-dimensional case for

$$
\begin{aligned}
f(q, x) & =\sum q^{\ell} C_{\ell}(x) \\
V(q) & =\sum_{\ell} V^{(\ell)} q^{\ell}
\end{aligned}
$$

becomes

$$
\Sigma_{m=0}^{\ell}\left\{(\ell+1-m)(m+1) C_{\ell+1-m} C_{m+1}-g(x) C_{\ell-m}^{\prime} C_{m}^{\prime}\right\}+V^{(\ell)}=0 .
$$

Now, taking

$$
C_{\ell}(x)=\Sigma_{0}^{\infty} b_{\ell m} x^{m} g(x)=\Sigma_{0}^{\infty} g_{n} x^{n},
$$

we find (for coefficients of $x^{r}$ ),

$r=0$ :

$$
\Sigma_{m=0}^{\ell}\left\{(\ell+1-m)(m+1) b_{\ell+1-m, 0} b_{m+1,0}-g_{0} b_{\ell-m, 1} b_{m, 1}\right\}+V^{(\ell)}=0
$$

and for

$r \geq 1$ :

$$
\begin{aligned}
& \sum_{m=0,0 \leq p \leq r}^{\ell}(\ell+1-m)(m+1) b_{\ell+1-m, p} b_{m+1, r-p} \\
& -\Sigma_{n, 1 \leq p \leq r+1}^{\ell} g_{n} b_{\ell-m, p} b_{m, r-n-p+2} \times p(r-n-p+2)=0 .
\end{aligned}
$$

Note that for the case $r \geq 1$, the potential does enter explicitly since it has no $x$ dependence. The relations Equations (54) and (55) provide the basis for a systematic recursion. 
One can easily work out several terms to see how the algorithm develops. It is clear that it is iteratively closed, but it is difficult to draw detailed conclusions on the solutions without extensive computations, as well as specification of potential models.

In the next section we give a proof in one dimension that the computation converges, with some reasonable assumptions. The method of proof can be generalized to $n$ dimensions.

\section{Convergence of the Algorithm in One Dimension}

Now, in Equation (52), define

$$
D_{m}=m C_{m},
$$

and note that the first term in Equation (52) can then be written as

$$
\sum_{m=0}^{\ell} D_{\ell+1-m} D_{m+1}=\Sigma_{m=1}^{\ell+1} D_{n} A^{(\ell)}{ }_{n m} D_{m},
$$

where symmetric the matrices $A^{(\ell)}{ }_{n m}$ consist of completely skew diagonal 1's, a reflection of the combinatorial origin of the coefficients. The trace is zero for even and unity for odd $\ell$ 's, and the eigenvalues are \pm 1 . They can occur in any order, but the orthogonal matrices that diagonalize $A^{(\ell)}$ may be constructed so that that the eigenvalues alternate (this is convenient for our proof of convergence but not necessary). Let us call these orthogonal matrices $u^{(\ell)}{ }_{n m}$ and represent the "vectors" $D_{m}$ in terms of the eigenvectors $d_{n}^{\ell}$ as

$$
D_{m}=\Sigma_{n=1}^{\ell+1} u^{(\ell)}{ }_{m n} d_{n}^{\ell},
$$

where

$$
\Sigma_{n=1}^{\ell+1} u_{m n}^{(\ell)} u^{(\ell)}{ }_{m^{\prime} n}=\delta_{m m^{\prime}}
$$

We then obtain

$$
\Sigma_{m=0}^{\ell} D_{\ell+1-m} D_{m+1}=\Sigma_{m=1}^{\ell+1} D_{n} A^{(\ell)}{ }_{n m} D_{m}=\Sigma_{m=1}^{\ell+1} \lambda^{(\ell)}{ }_{m}\left(d_{m}^{\ell}\right)^{2} .
$$

Now, consider the sum in the second term of Equation (52):

$$
\sum_{m=0}^{\ell} C_{\ell-m}^{\prime}(x) C_{m}^{\prime}(x)=\Sigma_{m=0}^{\ell} C_{m}^{\prime} B^{(\ell)}{ }_{m n} C_{n}^{\prime},
$$

where $B^{(\ell)}{ }_{m n}=A^{(\ell)}{ }_{m+1, n+1}$, the same set of matrices as $A^{(\ell)}$, occurring here with indices $1, \ldots \ell+1$ as well. By shifting the indices in the vectors $C_{n}^{\prime}$ by unity, one obtains the same structure as for the left hand side, i.e., for $m=0, \ldots \ell$, and $f$ the eigenvectors constructed from $C^{\prime}$,

$$
C_{m-1}^{\prime}=\Sigma_{n=1}^{\ell+1} u_{m n}^{(\ell)} f_{n}^{\ell} .
$$

We then have

$$
\sum_{m=0}^{\ell} C_{m}^{\prime} B^{(\ell)}{ }_{m n} C_{n}^{\prime}=\Sigma_{m=1}^{\ell+1} \lambda^{(\ell)}{ }_{m}\left(f_{m}{ }^{\ell}\right)^{2}
$$

so that our condition for a solution to Equation (52) becomes

$$
V^{(\ell)}+\Sigma_{m=1}^{\ell+1} \lambda_{m}^{(\ell)}\left[\left(d_{m}^{\ell}\right)^{2}-g(x)\left(f_{m}^{\ell}\right)^{2}\right]=0 .
$$

We now study the convergence of the $d$ and $f$ sums as $\ell \rightarrow \infty$. Inverting Equations (58) and (62), we obtain

$$
d_{m}^{\ell}=\Sigma_{n=1}^{\ell+1} n C_{n} u^{(\ell)}{ }_{n m}
$$

and

$$
f_{m}^{\ell}=\Sigma_{n=1}^{\ell+1} C_{n-1}^{\prime} u^{(\ell)}{ }_{n m}
$$


Since $u^{(\ell)}{ }_{n m}$ is an orthogonal matrix, it follows that

$$
\Sigma_{n=1}^{\ell+1}\left(f_{m}^{\ell}\right)^{2}=\Sigma_{n=1}^{\ell+1} C_{n-1}^{\prime 2}
$$

and

$$
\Sigma_{n=1}^{\ell+1}\left(d_{m}^{\ell}\right)^{2}=\Sigma_{n=1}^{\ell+1} n^{2} C_{n}^{2}
$$

It is sufficient to argue that the sequences in these sums are decreasing. The alternating (due to the $\lambda_{m}{ }^{\ell}$ ) series appearing in Equation (64) then converges.

We first remark that the generating function $f(q, X)$ is $C^{\infty}$ in both variables, so that all orders of derivatives with respect to $q$ exist. We seek solutions that can be represented as power series in $q$. Suppose that this series converges for all values of $q<q_{0}(x)$ (the radius of convergence can depend on $x$ ), and call $D_{\epsilon}$ the domain of $x$ such that $\left|q_{0}(x)\right| \geq \epsilon>0$, The ratio test prescribes that, for each such $x$,

$$
\left|\frac{C_{\ell+1}}{C_{\ell}}\right|<\frac{1}{\left|q_{0}(x)\right|}
$$

The series, Equation (46), corresponds to the Taylor expansion

$$
f(q, x)=\sum_{0}^{\infty} \frac{1}{\ell !} f^{\ell},
$$

where

$$
f^{\ell}=\frac{\partial^{\ell} f}{\partial q^{\ell}}
$$

The ratio condition then becomes

$$
\left|\frac{f^{\ell+1}}{f^{\ell}}\right|<\left|\frac{\ell+1}{q_{0}}\right| .
$$

If the derivatives do not grow faster than linearly, this condition should be satisfied for sufficiently large $\ell$. Taking $\left|q_{0}\right|=\epsilon$, the convergence would be uniform in $D_{\epsilon}$.

Now, consider the decreasing property. As for any series depending on a dimensional variable, we may scale the dimension, for $\left|q_{0}\right|>0$, so that $\left|q_{0}(x)\right|>1$ for all $x \in D_{\epsilon}$ (the ratio $C_{\ell+1} / C_{\ell}$ scales with $1 / q$ as well). This choice of scale is adequate for all $x \in D_{\epsilon}$ for a scale such that $\epsilon>1$. Then, uniformly, the $\left|C_{\ell}(x)\right|$ forms a decreasing sequence, leading to convergence of the $d$ series in Equation (64) (the factor $m$ in Equation (56) does not affect the convergence for large $m$ ). A similar argument can be followed for the $f$ series following the convergence of the series in $q$ for $\partial f(q, x) / \partial x$.

This completes our proof of convergence.

As remarked in the introduction, the nonliear expansions can be studied by means of Fourier series representations in terms of (upper half place) analytic functions (see, for example [18]), which may provide useful approximation techniques in specific cases. This study will appear in a later publication.

\section{Shift of Origin for Expansion}

We now return to arbitrary dimension. The algorithm proposed in Section 3 contains an expansion of the potential function $V(q)$ around some point $q=0$; for a polynomial potential or some other entire function, there would be no question of convergence of this expansion, but the algorithm itself may have only a finite domain of convergence. To extend the range of the resulting functions, it would then be necessary to carry out the expansions around some new origin at, e.g., $q=q_{0}$.

Therefore, let us now consider expanding $V(q)$ around $q_{0}$, and carry out the same procedure. We then rewrite Equation (25) for the modified problem with a new potential function

$$
V^{\prime}(q)=V\left(q+q_{0}\right)
$$


as

$$
V^{\prime}(q)+\delta_{i j} \frac{1}{2 m}\left(\frac{\partial \tilde{f}\left(q, x^{\prime}\right)}{\partial q_{i}}\right)\left(\frac{\partial \tilde{f}\left(q, x^{\prime}\right)}{\partial q_{j}}\right)=g_{i j}\left(x^{\prime}\right)\left(\frac{\partial \tilde{f}\left(q, x^{\prime}\right)}{\partial x_{i}^{\prime}}\right)\left(\frac{\partial \tilde{f}\left(q, x^{\prime}\right)}{\partial x_{j}^{\prime}}\right),
$$

where we observe that the solutions $\tilde{f}\left(q, x^{\prime}\right)$ and the manifold which we label $x^{\prime}$ will be different from $f(q, x)$ on the manifold $x$ since the potential function $V^{\prime}(q)$ is different; however, the variable $q$ on the original space is still designated by $q$ since it is the argument of $V^{\prime}(q)$.

The assumptions underlying Equation (74) imply that in the generating function $\tilde{f}\left(q, x^{\prime}\right), q$ and $x^{\prime}$ are independant variables; we may then proceed by recognizing that, as a result of the solution algorithm, $x^{\prime}$ can only be a function of $x$ in the mapping $q, x \rightarrow q, x^{\prime}$.

We can now use the chain rule of derivatives for the right hand side and consider $\tilde{f}\left(q, x^{\prime}\right)$ as a function of $q, x$, at least locally under this map. Calling this function $h\left(q+q_{0}, x\right)$, we can rewrite Equation (74) as

$$
V^{\prime}(q)+\delta_{i j} \frac{1}{2 m} \frac{\partial h\left(q+q_{0}, x\right)}{\partial q_{i}} \frac{\partial h\left(q+q_{0}, x\right)}{\partial q_{j}}=\tilde{g}_{i j}(x) \frac{\partial h\left(q+q_{0}, x\right)}{\partial x_{i}} \frac{\partial h\left(q+q_{0}, x\right)}{\partial x_{j}},
$$

where

$$
\tilde{g}_{i j}(x)=g_{k \ell}\left(x^{\prime}\right) \frac{\partial x_{i}}{\partial x_{k}^{\prime}} \frac{\partial x_{j}}{\partial x_{\ell}^{\prime}} .
$$

Replacing as a change of variables $q+q_{0} \rightarrow q, V^{\prime}(q)$ becomes $V(q)$, and Equation (75) becomes

$$
V(q)+\delta_{i j} \frac{1}{2 m} \frac{\partial h(q, x)}{\partial q_{i}} \frac{\partial h(q, x)}{\partial q_{j}}=\tilde{g}_{i j}(x) \frac{\partial h(q, x)}{\partial x_{i}} \frac{\partial h(q, x)}{\partial x_{j}}
$$

Since this equation has a solution (among others) of the form for which

$$
\tilde{g}_{i j}(x)=g_{i j}(x),
$$

by applying the same algorithm, we may choose this solution with the consequence that

$$
g_{k \ell}\left(x^{\prime}\right) \frac{\partial x_{i}}{\partial x_{k}^{\prime}} \frac{\partial x_{j}}{\partial x_{\ell}^{\prime}}=g_{i j}(x) .
$$

With this choice we may follow shifts from $q \rightarrow q_{0} \rightarrow q_{1} \ldots$ within the domains of convergence choosing the same algorithm for solution at every step, building a set of overlapping neighborhoods that construct a manifold, on which covariance is maintained through the canonical transformation.

\section{Change in Generating Function Induced by Diffeomorphisms in the Geometric Space}

The structure of the image space has the property of supporting local diffeomorphisms. However, our construction concerns a mapping from the the coordinates $\{q, p\}$ to $\{x, \pi\}$; therefore, a diffeomorphism of the latter set of variables necessarily involves a change in the generating function of the transformation.

In this section, we calculate the effect of an infinitesimal coordinate transformation on the geometrical space, holding the Hamiltonian variables $\{q, p\}$ unchanged, on the generating function of the canonical transformation, i.e., $f \rightarrow \tilde{f}$.

On the original choice of coordinates, for which

$$
\begin{aligned}
p_{i} & =\frac{\partial f(q, x)}{\partial q_{i}} \\
\pi_{i} & =-\frac{\partial f(q, x)}{\partial x_{i}}
\end{aligned}
$$


we now consider a new mapping from $q, p$ to $x^{\prime}, \pi^{\prime}$ differing infinitesimally from $x, \pi$ according to

$$
x_{i}^{\prime}=x_{i}+\lambda_{i}(x)
$$

where $\lambda_{i}(x)$ is small.

After this mapping, we can write

$$
\begin{aligned}
& p_{i}=\frac{\partial \tilde{f}\left(q, x^{\prime}\right)}{\partial q_{i}} \\
& \pi_{i}^{\prime}=-\frac{\partial \tilde{f}\left(q, x^{\prime}\right)}{\partial x_{i}^{\prime}} .
\end{aligned}
$$

To study $\tilde{f}\left(q, x^{\prime}\right)$, let us define

$$
g^{i}\left(q, x^{\prime}\right)=\frac{\partial \tilde{f}\left(q, x^{\prime}\right)}{\partial x_{i}^{\prime}}=-\pi_{i}^{\prime}
$$

Then,

$$
g^{i}(q, x+\lambda) \cong \frac{\partial \tilde{f}(q, x)}{\partial x_{i}}+\frac{\partial^{2} \tilde{f}(q, x)}{\partial x_{i} \partial x_{j}} \lambda_{j}(x)
$$

so that

$$
-\pi^{\prime i} \cong \frac{\partial \tilde{f}(q, x)}{\partial x_{i}}+\frac{\partial^{2} \tilde{f}(q, x)}{\partial x_{i} \partial x_{j}} \lambda_{j}(x) .
$$

This result could have been obtained directly from Equation (82) but it is perhaps helpful to define the function $g^{i}\left(q, x^{\prime}\right)$ to clarify the computation.

We now impose invariance of

$$
\pi^{\prime i} d x_{i}^{\prime}=\pi^{i} d x_{i}
$$

which leads, through the Hamilton-Lagrange construction, to invariance of the Hamiltonian. We now write out

$$
\begin{aligned}
-\pi^{\prime i} d x_{i}^{\prime} \cong\left[\frac{\partial \tilde{f}(q, x)}{\partial x_{i}}\right. & \left.+\frac{\partial^{2} \tilde{f}(q, x)}{\partial x_{i} \partial x_{j}} \lambda_{j}(x)\right] \times\left[d x_{i}+\frac{\partial \lambda_{i}}{\partial x_{k}} d x_{k}\right]= \\
& =\frac{\partial \tilde{f}(q, x)}{\partial x_{i}} d x_{i}+\frac{\partial^{2} \tilde{f}(q, x)}{\partial x_{i} \partial x_{j}} \lambda_{j}(x) d x_{i}= \\
& +\frac{\partial \tilde{f}(q, x)}{\partial x_{i}} \frac{\partial \lambda_{i}}{\partial x_{k}} d x_{k}+\frac{\partial^{2} \tilde{f}(q, x)}{\partial x_{i} \partial x_{j}} \lambda_{j}(x) \frac{\partial \lambda_{i}}{\partial x_{k}} d x_{k}=-\pi^{i} d x_{i} .
\end{aligned}
$$

Therefore, to order $\lambda d x$,

$$
\begin{aligned}
d x_{i} \frac{\partial f(q, x)}{\partial x_{i}} & =d x_{i}\left\{\frac{\partial \tilde{f}(q, x)}{\partial x_{i}}+\frac{\partial^{2} \tilde{f}(q, x)}{\partial x_{i} \partial x_{j}} \lambda_{j}(x)+\frac{\partial \tilde{f}(q, x)}{\partial x_{k}} \frac{\partial \lambda_{k}}{\partial x_{i}}\right\}= \\
& =d x_{i}\left\{\frac{\partial \tilde{f}(q, x)}{\partial x_{i}}+\frac{\partial}{\partial x_{i}}\left[\frac{\partial \tilde{f}(q, x)}{\partial x_{k}} \lambda_{k}\right]\right\}
\end{aligned}
$$

so that

$$
d x_{i} \frac{\partial f(q, x)}{\partial x_{i}}=d x_{i} \frac{\partial}{\partial x_{i}}\left[\tilde{f}(q, x)+\lambda_{k} \frac{\partial \tilde{f}(q, x)}{\partial x_{k}}\right] .
$$

If we write (say, integrate up to some $x_{i}$ )

$$
f(q, x)=\tilde{f}(q, x)+\lambda_{k} \frac{\partial \tilde{f}(q, x)}{\partial x_{k}}
$$


we may approximately invert to get

$$
\tilde{f}(q, x) \cong f(q, x)-\lambda_{k} \frac{\partial f(q, x)}{\partial x_{k}} .
$$

This corresponds to a conformal-like local transformation. The algebra of such generators is

$$
\left[\lambda_{i}^{a} \frac{\partial}{\partial x_{i}}, \lambda_{j}^{b} \frac{\partial}{\partial x_{j}}\right]=\left(\lambda_{i}^{a} \frac{\partial \lambda_{j}^{b}}{\partial x_{i}}-\lambda_{i}^{b} \frac{\partial \lambda_{j}^{a}}{\partial x_{i}}\right) \frac{\partial}{\partial x_{j}} .
$$

Thus the algebra is of a conformal type, but the coefficients may run on, so that the group may not be finite-dimensional.

Example: Suppose $\lambda_{i}{ }^{a}=\epsilon_{i}{ }^{j}(a) x_{j}$, such as a rotation generator (we may factor out the infinitesimal scale), for $\epsilon_{i}{ }^{j}(a)$ antisymmetric constants. Then,

$$
\left[\lambda_{i}^{a} \frac{\partial}{\partial x_{i}}, \lambda_{j}^{b} \frac{\partial}{\partial x_{j}}\right]=x_{j} M_{i}^{j}(b, a) \frac{\partial}{\partial x_{i}},
$$

where

$$
M_{i}{ }^{j}(b, a)=\epsilon_{i}{ }^{k}(b) \epsilon_{k}{ }^{j}(a)-\epsilon_{i}{ }^{k}(a) \epsilon_{k}{ }^{j}(b) .
$$

For the rotation group, these form a finite Lie algebra. The group acts on the generating function (which forms a representation) but does not affect the $\{q, p\}$ variables.

\section{One-Dimensional Conformal Metric}

In this section we study the important case of the conformal metric in the image of our canonical transformation and, in particular, the illustrative example of the harmonic oscillator potential in one dimension. Higher-dimensional examples can be treated using similar methods.

Since the transformation establishes a bijective correspondence between the orbits induced by the original Hamiltonian and those induced by the geometrical form, we understand that the Hamiltonian coordinate $q$ can be considered a function of the corresponding geometric coordinate $x$ (argued from a different point of view in Ref. [11]). For the sake of definiteness, and for its relevance to the work of Ref. [1], we shall fix the metric to be of the form

$$
g(x)=\frac{E}{E-V(x)}
$$

where $E$ is a parameter identified with the energy surface on which the motion takes place. The form of the metric in Equation (95) was chosen ad hoc in reference [1] since it provided a simple formal link between the two forms of the generators of motion. It has been an outstanding question of why the local stability properties of the geometric form of the mechanics, as measured through geodesic deviation, should be in agreement with the stability properties of the orbits generated by the original Hamiltonian. The existence of the canonical transformation between the two forms of dymanics, due to its injective nature, answers this question. We show in this section that a generating function for the transformation exists, and how it can be constructed for the simple case of the harmonic oscillator.

Consider again Equation (50) for the generating function in one dimension. Assuming that the potential $V(q)$ is positive we get that $g(x)>0$. In this case we can write Equation (50) in the form

$$
\left(\frac{\partial f}{\partial q}(q, x)\right)^{2}+2 m V(q)=\left(\sqrt{g(x)} \frac{\partial f}{\partial x}(q, x)\right)^{2}
$$


If we define a new independent variable $x^{\prime}$ by setting

$$
\frac{d x}{d x^{\prime}}\left(x^{\prime}\right)=\sqrt{g(x)}
$$

we obtain

$$
\left(\frac{\partial f}{\partial q}\right)^{2}+2 m V(q)=\left(\frac{d x}{d x^{\prime}} \frac{\partial f}{\partial x}\right)^{2}
$$

We shall assume that Equation (97) can be integrated to obtain an invertible function $x=x\left(x^{\prime}\right)$. Now define a new function $f_{1}\left(q, x^{\prime}\right)$ by

$$
f_{1}\left(q, x^{\prime}\right)=f\left(q, x\left(x^{\prime}\right)\right) .
$$

In this case we have

$$
\frac{\partial f_{1}}{\partial q}\left(q, x^{\prime}\right)=\frac{\partial f}{\partial q}(q, x), \quad \frac{\partial f_{1}}{\partial x^{\prime}}\left(q, x^{\prime}\right)=\frac{d x}{d x^{\prime}} \frac{\partial f}{\partial x}(q, x)=\sqrt{g(x)} \frac{\partial f}{\partial x}(q, x)
$$

so that Equation (98) can be written in the form

$$
\left(\frac{\partial f_{1}}{\partial q}\right)^{2}+2 m V(q)=\left(\frac{\partial f_{1}}{\partial x^{\prime}}\right)^{2}
$$

If we can solve Equation (101) for $f_{1}\left(q, x^{\prime}\right)$ we may obtain the generating function $f(q, x)$ by $f(q, x)=f_{1}\left(q, x\left(x^{\prime}\right)\right)$. Observe that the generating function $f(q, x)$ obtained in this way incorporates the, as yet undetermined, metric $g(x)$ through Equation (97).

Let us try to solve Equation (101), or equivalently,

$$
2 m V(q)=\left(\frac{\partial f_{1}}{\partial x^{\prime}}\right)^{2}-\left(\frac{\partial f_{1}}{\partial q}\right)^{2}
$$

Introducing new independent variables $y_{1}, y_{2}$ by

$$
q=y_{1}-y_{2}, \quad x^{\prime}=y_{1}+y_{2},
$$

and setting

$$
f_{2}\left(y_{1}, y_{2}\right)=f_{1}\left(q\left(y_{1}, y_{2}\right), x^{\prime}\left(y_{1}, y_{2}\right)\right)
$$

we have

$$
\frac{\partial}{\partial y_{1}}=\frac{\partial x^{\prime}}{\partial y_{1}} \frac{\partial}{\partial x^{\prime}}+\frac{\partial q}{\partial y_{1}} \frac{\partial}{\partial q}=\frac{\partial}{\partial x^{\prime}}-\frac{\partial}{\partial q} \quad \frac{\partial}{\partial y_{2}}=\frac{\partial x^{\prime}}{\partial y_{2}} \frac{\partial}{\partial x^{\prime}}+\frac{\partial q}{\partial y_{2}} \frac{\partial}{\partial q}=\frac{\partial}{\partial x^{\prime}}+\frac{\partial}{\partial q}
$$

and hence Equation (102) becomes

$$
\frac{\partial f_{2}}{\partial y_{2}} \cdot \frac{\partial f_{2}}{\partial y_{1}}=2 m V\left(y_{1}-y_{2}\right)
$$

As an example, let us solve Equation (103) for the case of the harmonic oscillator potential

$$
V(q)=\frac{1}{2} m \omega^{2} q^{2}
$$


Equation (103) becomes in this case

$$
\frac{\partial f_{2}}{\partial y_{2}} \cdot \frac{\partial f_{2}}{\partial y_{1}}=m^{2} \omega^{2}\left(y_{1}-y_{2}\right)^{2}
$$

To solve Equation (105) we expand $f_{2}\left(y_{1}, y_{2}\right)$ into a power series in $y_{1}, y_{2}$,

$$
\begin{aligned}
f_{2}\left(y_{1}, y_{2}\right)=p_{0}\left(c_{1} y_{1}+c_{2} y_{2}\right)+\frac{p_{0}}{L_{0}}\left(c_{3} y_{1}^{2}+\right. & \left.c_{4} y_{1} y_{2}+c_{5} y_{2}^{2}\right)+\frac{p_{0}}{L_{0}^{2}}\left(c_{6} y_{1}^{3}+c_{7} y_{1}^{2} y_{2}+c_{8} y_{1} y_{2}^{2}+c_{9} y_{2}^{3}\right) \\
& +\frac{p_{0}}{L_{0}^{3}}\left(c_{10} y_{1}^{4}+c_{11} y_{1}^{3} y_{2}+c_{12} y_{1}^{2} y_{2}^{2}+c_{13} y_{1} y_{2}^{3}+c_{14} y_{2}^{4}\right)+\cdots
\end{aligned}
$$

with the constants, $p_{0}$ with dimension of momentum and $L_{0}$ with dimension of length, inserted in order for the unknown coefficients to be dimensionless. Next, we insert this expansion into Equation (105) and obtain equations for the coefficients. One possible solution of this set of equations is (we observe that the number of undetermined coefficients is greater than the number of equations we obtain so that there is a certain freedom in choosing the values of the coefficients),

$$
f_{2}\left(y_{1}, y_{2}\right)=p_{0} y_{1}+\frac{p_{0}}{L_{0}} y_{1}^{2}+\frac{p_{0}}{L_{0}^{2}} \alpha\left(y_{1}^{2} y_{2}-y_{1} y_{2}^{2}+\frac{1}{3} y_{2}^{3}\right)+\frac{p_{0}}{L_{0}^{3}} \alpha\left(-2 y_{1}^{3} y_{2}+2 y_{1}^{2} y_{2}^{2}-\frac{2}{3} y_{1} y_{2}^{3}\right)+\cdots
$$

where $\alpha=L_{0}^{2} m^{2} \omega^{2} / p_{0}^{2}$. Changing variables back to $q, x^{\prime}$ we obtain

$$
\begin{aligned}
f_{1}\left(q, x^{\prime}\right) & =f_{2}\left(y_{1}\left(q, x^{\prime}\right), y_{2}\left(q, x^{\prime}\right)=p_{0} \frac{1}{2}\left(x^{\prime}+q\right)\right. \\
& +\frac{p_{0}}{L_{0}} \frac{1}{4}\left(x^{\prime}+q\right)^{2}+\frac{p_{0}}{L_{0}^{2}} \alpha\left(\frac{1}{8}\left(x^{\prime 2}-q^{2}\right) q+\frac{1}{24}\left(x^{\prime}-q\right)^{3}\right) \\
& +\frac{p_{0}}{L_{0}^{3}} \alpha\left(-\frac{1}{8}\left(x^{\prime}+q\right)^{2}\left(x^{\prime 2}-q^{2}\right)+\frac{1}{8}\left(x^{\prime 2}-q^{2}\right)^{2}-\frac{1}{24}\left(x^{\prime 2}-q^{2}\right)\left(x^{\prime}-q\right)^{2}\right)+\cdots .
\end{aligned}
$$

We turn now to find the dependence $x^{\prime}=x^{\prime}(x)$ by integration of Equation (97). We have

$$
\frac{d x}{d x^{\prime}}\left(x^{\prime}\right)=\sqrt{g(x)}=\sqrt{\frac{E}{E-V(x)}} \Rightarrow \frac{d x^{\prime}}{d x}(x)=\sqrt{\frac{E-V(x)}{E}} .
$$

Hence, we may define

$$
x^{\prime}(x)=\int_{0}^{x} \sqrt{1-\frac{1}{E} V(t)} d t .
$$

In particular, in the case of the harmonic oscillator we have

$$
\begin{aligned}
x^{\prime}(x) & =\int_{0}^{x} \sqrt{1-\frac{1}{E} V(t)} d t=x^{\prime}(x)=\int_{0}^{x} \sqrt{1-\frac{m \omega^{2}}{2 E} t^{2}} d t= \\
& =\sqrt{\frac{E}{2 m \omega^{2}}}\left[\arcsin \left(\sqrt{\frac{m \omega^{2}}{2 E}} x\right)+\frac{1}{2} \sin \left(2 \arcsin \left(\sqrt{\frac{m \omega^{2}}{2 E} x}\right)\right)\right] .
\end{aligned}
$$

Once the function $x^{\prime}=x^{\prime}(x)$ is determined we obtain the generating function $f(q, x)$ by recalling that $f(q, x)=f_{1}\left(q, x^{\prime}(x)\right)$. In particular, for $|x|<<1$ we get from Equation (109) that

$$
x^{\prime}=x-\frac{1}{6} \cdot \frac{m \omega^{2}}{2 E} x^{3}+\cdots
$$


and if we plug this into Equation (107) we get

$$
\begin{aligned}
f(q, x) & =f_{1}\left(q, x^{\prime}(x)\right)= \\
& =p_{0} \cdot \frac{1}{2}(q+x)+\frac{p_{0}}{L_{0}} \cdot \frac{1}{4}(q+x)^{2}+\frac{p_{0}}{L_{0}^{2}} \alpha\left(-\frac{1}{6} q^{3}+\frac{1}{8} q^{2} x+\left(\frac{p_{0}^{2}}{12 m E}+\frac{1}{24}\right) x^{3}\right)+\cdots .
\end{aligned}
$$

Equation (110) provides the first few terms in a series expansion of a generating function for a symplectomorphism from standard Hamiltonian dynamics to the geometrical form with metric given by Equation (95).

\section{Mapping of Bounded Submanifolds}

Since the mapping that we have constructed carries a Euclidean phase space into a geometrical form, it is natural to study possibly non-trivial topological properties that this geometrical space could have. As a simple example, consider a potential in the Euclidean space in two dimensions which contains two identical finite depth potential wells with lower bound $E_{0}$, and centers spaced along the $x$-axis. Above a certain energy, say $E_{1}$, there is just one connected region of motion, and between $E_{1}$ and $E_{0}$ there are two separated regions. The total energy serves as a hight function, in the terminology of Morse theory [20] (see also [19]).

Let us first consider a particle with energy $E_{0}<E<E_{1}$. A particle in one of these wells has an orbit that is confined to this well. If it reaches the boundary where $E=V$, the momentum (and velocity) vanishes, and the orbit necessarily then retraces its path as under time reversal. Under the symplectomorphism, this orbit is mapped into a geodesic curve, and by the property of $1: 1$ mapping, the corresponding geodesic curve must stop and retrace its path as under time reversal as well. The family of all such orbits for a given value of $E$ defines a boundary in the geometric space, and is therefore a closed submanifold with boundary.

It is clear that such orbits associated with each well (at a given value of $E$ ) separately are disjoint since they are disjoint in the original space. Increasing the energy above the value $E_{1}$ would result in a single connected region for the geometric orbits. Therefore the homotopy classes of the possible orbits change as a function of the height function $E$. We shall explore the consequences, in particular, of the existence of topoligical invariants, in this context in a later publication.

\section{Summary and Conclusions}

In this paper we have constructed a canonical transformation from a Hamiltonian of the usual form given in Equation (1) to a geometric form of Equation (2).

We have given the basic mathematical formulation in terms of the geometry of symplectic manifolds.

For the central purpose of our construction, we formulate the process of studying stability by means of geodesic deviation in terms of geometric methods, making clear the relation between stability in the geometric manifold and the original Hamiltonian motion.

We then give an algorithm for solving the nonlinear equations for the generating function of the canonical transformation. This algorithm was then studied for the simple case of one dimension, and we proved convergence of the recursive scheme under certain reasonable assumptions.

Since the series expansions generated by the algorithm for finding the solutions for the generating function may have a bounded domain of convergence, we studied (in general dimensions) the possibility of shifting the origin in order to carry out the expansions based on a new origin. As for the analytic continuation of a function of a complex variable, this procedure can extend the solutions for the generating function to a maximal domain.

Since the image space of the symplectomorphism has geometrical structure, it is natural to study its properties under local diffeomorphisms. A local change of variables $\{x, \pi\} \rightarrow\left\{x^{\prime}, \pi^{\prime}\right\}$ (leaving the variables of the original space unchanged) alters the structure of the mapping from the original 
variables $\{q, p\}$ to the new variables $\left\{x^{\prime}, \pi^{\prime}\right\}$; we study the effect of infinitesinal diffeomorphims of this type on the generating function.

We finally discussed briefly the mapping of bounded closed submanifolds, created by potential wells in the Hamiltonian space, corresponding to closed submanifolds in the geometric space, where Morse theory may be applied, to open the possibility of obtaining a new class of conserved quantities associated with homotopies of the image space.

Author Contributions: Conceptualization, L.P.H., Y.S., J.L. and A.Y.; methodology, L.P.H. and Y.S.; software, Y.S.; validation, L.P.H. and Y.S.; formal analysis, Y.S.; investigation, L.P.H., Y.S., J.L. and A.Y.; resources, L.P.H., J.L. and A.Y.; writing - original draft preparation, L.P.H. and Y.S.; writing-review and editing, L.P.H. and Y.S.; supervision, L.P.H., Y.S., J.L. and A.Y.; project administration, L.P.H. and A.Y.; funding acquisition, L.P.H., J.L. and A.Y.; All authors have read and agreed to the published version of the manuscript.

Funding: This research received no external funding.

Acknowledgments: We thank Gil Elgressy and Michal Wagman for discussions.

Conflicts of Interest: Authors declare no conflict of interest.

\section{References}

1. Horwitz, L.; Zion, Y.B.; Lewkowicz, M.; Schiffer, M.; Levitan, J. Geometry of Hamiltonian chaos. Phys. Rev. Lett. 2007, 98, 234301. [CrossRef] [PubMed]

2. Pettini, M. Geometry and Topology in Hamiltonian Dynamics and Statistical Mechanics; Springer: New York, NY, USA, 2007.

3. Arnold, V.I. Mathematical Methods of Classical Mechanics; Springer: New York, NY, USA, 1978.

4. Guckenheimer, J.; Holmes, P. Nonlinear Oscillations, Dynamical Systems, and Bifurcations of Vector Fields; Springer: New York, NY, USA, 1983.

5. DiBenedetto, E. Classical Mechanics, Theory and Mathematical Modelling; Springer: New York, NY, USA, 2011.

6. Alligood, K.T.; Sauer, T.D.; Yorke, J.A. Chaos, and Introduction to Dynamical Systems; Springer: New York, NY, USA, 1996.

7. Gutzwiller, M.C. Chaos in Classical and Quantum Mechanics; Springer: New York, NY, USA, 1990.

8. Curtis, W.D.; Miller, F.R. Differentiable Manifolds and Theoretical Physics; Academic Press: New York, NY, USA, 1985.

9. Weinberg, S. Gravitation and Cosmology: Principles and Applications of the General Theory of Relativity; John Wiley and Sons: New York, NY, USA, 1972.

10. Zion, Y.B.; Horwitz, L. Detecting order and chaos in three dimensional Hamiltonian systems by geometrical methods. Phys. Rev. E 2007, 76, 046220. [CrossRef] [PubMed]

11. Zion, Y.B.; Horwitz, L. Applications of geometrical criteria for transition to Hamiltonian chaos. Phys. Rev. E 2007, 78, 036209. [CrossRef] [PubMed]

12. Zion, Y.B.; Horwitz, L. Controlling effect of geometrically defined local structural changes on chaotic systems. Phys. Rev. E 2010, 81, 046217. [CrossRef] [PubMed]

13. Lewkowicz, M.; Levitan, J.; Zion, Y.B.; Horwitz, L.P. Geometry of Local Instability in Hamiltonian Dynamics. In Handbook of Applications of Chaos Theory; Chapman and Hall, CRC Press: New York, NY, USA, 2016; Chapter. 15, pp. 231-252.

14. Horwitz, L.P.; Yahalom, A.; Levitan, J.; Lewkowicz, M. An underlying geometrical manifold for Hamiltonian mechanics. Front. Phys. 2016, 12, 124501. [CrossRef]

15. Calderon, E.; Horwitz, L.; Kupferman, R.; Shnider, S. On the geometric formulation of Hamiltonian dynamics. Chaos 2013, 23, 013120. [CrossRef] [PubMed]

16. Da Silva, A.C.; Da Salva, A.C. Lectures on Sympletic Geometry; Lecture Notes in Mathematics 1764; Springer: New York, NY, USA, 2006.

17. Strauss, Y.; Horwitz, L.P.; Levitan, J.; Yahalom, A. Quantum field theory of Hamiltonian chaos. J. Math. Phys. 2015, 56, 072701. [CrossRef]

18. Hille, E. Ordinary Differential Equations in the Complex Plane; John Wiley and Sons: New York, NY, USA, 1976. 
19. Frankel, T. The Geometry of Physics. An Introduction; Cambridge University Press: Cambridge, UK, 1997.

20. Milnor, J. Morse Theory; Annals of Mathematics Studies 51; Princeton University Press: Princeton, NJ, USA, 1969.

(c)

(C) 2020 by the authors. Licensee MDPI, Basel, Switzerland. This article is an open access article distributed under the terms and conditions of the Creative Commons Attribution (CC BY) license (http://creativecommons.org/licenses/by/4.0/). 